\title{
Designing Context-Aware Interactions for Task-Based Applications*
}

\author{
Pablo Muñoz, Pau Giner, and Miriam Gil \\ Centro de Investigación en Métodos de Producción de Software \\ Universidad Politécnica de Valencia, 46022 Valencia, España \\ \{pmunoz,pginer,mgil\} @pros.upv.es
}

\begin{abstract}
Since contextual information has potential to improve task-based applications, we provide an approach for integrating contextual information in task-based applications by considering simplicity as a major design goal. We present a context-aware application to support mobile workflows focusing our solutions in three important factors to organize and manage tasks: priority, location and time. Following the simplicity guidelines, we provide solutions based on these factors by means of visualizations that allow users to complete their tasks fluently on the go.
\end{abstract}

Keywords: context-aware, interaction, location-based services, mobile workflow applications, mobile devices.

\section{Introduction}

The increasing improvement in the communication ability of mobile devices allows users to access information and web services anywhere and anytime. Furthermore, these devices have become full of sensors that can access contextual information such as the current location of the user by means of GPS coordinates. By improving the access to this contextual information, we increase the richness of communicationin human-computer interaction and make it possible to produce more usefulcomputational services [10]. One of these services could allow making daily activities more fluent through reducing the need for data input.

In particular, this work is focused on task-based applications. According to the classification of web applications defined by Unger in [8], task-based applications are defined as " a tool or collection of tools meant to allow users to accomplish a set of key tasks or workflows". As we have experienced in the development of Presto [1] contextual information has potential to improve task-based applications. This work extends the architecture components defined in Presto with interaction mechanisms to allow users to interact with the system taking into account different kinds of context information.

Interaction aspects are a crucial factor for determining the application's success or failure since a bad interaction can cause that user does not use the application.

* This work has been developed with the support of MICINN under the project SESAMO TIN2007-62894 and co-financed with ERDF. 
Specially, we have put emphasis in simplicity as one of the main goals of our design solutions. Some products such as iPod, which wasdesigned following a simplicity approach, has found success with a simple and non-intrusive design that does not invade the user's mind [5].

In this work we provide an approach for integrating contextual information in task-based applications by considering simplicity as a major design goal. In order to illustrate our approach we present a context-aware application to support mobile workflows. This application is in a prototypical stage but allows users to manage a todo list in a different way according to context conditions. The user can discover what to do next by organizing their tasks according to different context conditions such as location, time or priority. Context information has been integrated following the simplicity principles allowing users to complete their tasks fluently.

The remainder of this paper is structured as follows. Section 2 relates the interaction design process applied in our approach. Section 3 introduces the application and its proposals for adapting it to the user context. Section 4 introduces related work in the area. Finally, Section 5 concludes the paper.

\section{The Interaction Design Process}

For the design of context-aware applications we prioritized simplicity since we wanted to focus on the task at hand without any user distractions. We avoid complexity as much as possiblebecause it involvesa high mental effort and forces the user to divert his attention from other tasks.

The problems presented by complexity are usually accentuated due to the constraints introduced by mobile devices in terms of interaction (e.g., small screens, reduced processing power, etc.). For this reason, the idea of achieving a simple interaction design is essential to get and keep success in an application or product. However, designers find hard to create simple interaction designs that cover all requirements because there is no process or method to support the design of simple interfaces.

Since we wanted to obtain simple designs in an early step we inspired in the "Laws of Simplicity" of Maeda [5] in order to design our interactions (see table 1). These guidelines are metaphors which are applicable to different areas such asdesign, business, technology and life. In our work we took some of these guidelines to detect and fix complexity problems. Specifically, in our work we apply the laws:

- Reduce. To preserve the most relevant information and operations in the main screen, diverting the rest to other contexts where user can navigate.

- Organize. To group similar components and operations.

- Time. To avoid unnecessary navigation.

- Learn. To apply popular and intuitive knowledge that is movable to an application

- Context. To emphasize the more relevant factors according to the user needs.

According to these laws, we refined our interaction designs in different iterations in which new ideas are emerged such as contextualize the zoom controls. At the end, we obtained interaction designs more focused in the end-user. 
Table 1. Maeda's Laws of Simplicity

\begin{tabular}{ll}
\hline Law & Description \\
\hline 1 Reduce & The simplest way to achieve simplicity is through \\
& thoughtful reduction. \\
2 Organize & Organization makes a system of many appear fewer. \\
3 Time & Savings in time feel like simplicity. \\
4 Learn & Knowledge makes everything simpler. \\
5 Differences & Simplicity and complexity need each other. \\
6 Context & What lies in the periphery of simplicity is definitely \\
& not peripheral. \\
8 Emotion & More emotions are better than less. \\
9 Failure & In simplicity we trust. \\
10 The one & Some things can never be made simple. \\
& Simplicity is about subtracting the obvious, and adding \\
\end{tabular}

\section{The Application}

In a mobile environment where this work is situated, the appropriate information provided by the device is determined by the changes caused in the context of use (e.g., changes in location, in time, in activity, and more). Some of these changes, specially"location" and "time", were appointed as important types of context in [10].

Therefore, in the mentioned interaction design process, we considered the user's needs in different contexts by analyzing the typical patterns that users follow for completing their tasks. For this, in a brainstorming the authors analyzed the different features, some of them included in several to-do applications such as GeoLife, Remember the Milk and Astrid. Thus, we defined some possible requirements for the application. These possible requirements were: "the necessity of view the current tasks, identify tasks by priority criteria, know how arrive to the place where the task must be performed, view a general state of the agenda in just a screen, generate an optimized route, delegate tasks, establish order between task and support collaborative tasks, etc.". After the brainstorming session we determined that priority, location and time were the main factors for task organization and we limited application's functionality through selecting some of these requirements that were related to these factors.

Faced with these established necessities, we proposed solutions by means of an Android ${ }^{1}$-based prototype that provides three different visualizations. Each of thesevisualizations is focused on addressing the requirements detected for managing tasks in a particular context. Since users can move from view to view, they allow an adaptation to the user's current context of use. The three proposed visualizations are:

- Map View. It provides a view where the tasks are geo-located in a map, allowing the user to know in which locations has more pending tasks. In this way, user is urged to plan his optimized routes. This view shows also the user's current location, and it is centered on this context information.

- Timeline View. It provides a guided view over the progress of the tasks in time. Its objective is informing the user of the next tasks that must be performed with

\footnotetext{
${ }^{1}$ http://www.android.com/
} 
its associated cost. Similar to the Map View, this view is focused on time factor, since it is the most important attribute to take into account. So, we center the Timeline View in the next task that must be performed. In the same way that Map View centers his view on the most view's important attribute, location, Timeline View centers its view in its most important attribute, time, so we center its view in the next task that must be performed.

- Cloud View. It responds to the need to prioritize tasks. With the Cloud View, users get a high-general view of a set of tasks organized by priorities. In this way, they obtain a general view of its agenda in just a screen. The visualization consists in showing the tasks like a cloud of tags, where each tag is the name of a task and where each tag has a particular font according to its priority.

\subsection{Common Matters}

Although the proposed visualizations are different from each other, there are some common matters between them that we relate below:

General Interaction Concerns. Regarding to the general interaction we maintain two common interaction controls in all the visualizations. Thus, we applied the forth law of simplicity "learn" because the common use of the same controls in all of the visualizations reduces the mental effort for learning how to use the application. These interaction controls are filters that allow focusing the user's view in a smaller set of tasks (this idea applies the first law "reduce" in order to hide non-relevant data through the removal of far future or low-priority tasks).More specially, these filters are a time filter and a priority filter (see figure 1). The time filter allows showing tasks situated in a different ranges of time, which they are: "today, next three days, next week, next month, all time, and tasks that are not programmed for a concrete time (e.g. clean the basement)". Besides, the priority filter allows showing tasks with specific priority types, which these priority types are: "high, medium and low". Thus, the possible total combinations between priority and time values cover much of the user's needs for filtering tasks according to time and priority factors. For example, one of these possible combinations should be "gettingonlyhi priority tasks for the next week".

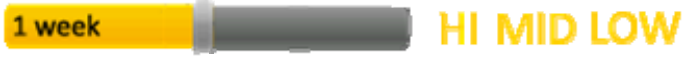

Fig. 1. Filter controls

Mechanism for switching visualization and adaptation of the visualization. According to the changes in user's context and applying the sixth law of simplicity "context", we define mechanisms that allow a change between visualizations in order to cover the current context needs. Furthermore, when the visualization is changed, another factor like the filters' state of the selection of a task should not be changed. So, we decide preserve these factors over context changes and focus the new visualization on the selected task and preserving the filters' state. Thus, we do not change the current user environment unexpectedly. 
Moreover, the current time and location are context information that is relevant for change dynamically the view. An automated change in view is only enabled when user is not interacting with the application. This generates good emotions in user (we apply here the seventh law of simplicity: "emotion") according to an underlying received data (such as higher proximity to a location where user has a pending task). We take advantage of this by means of the interpretation of the changes in location and time, two of the three main factors for task organization. So, when user is using any visualization and changes its location by other nearly where it has pending tasks, the application changes to the Map View automatically and sends a notification to the user. Besides, when the next task must soon be performed, the application changes automatically to the Timeline View and sends a notification to the user.

Task's interaction. Although one of the main goals of a task-based application is to provide users with a well-organized list of tasks, mechanisms are also required for handling this information. Thus, in our application we considered the operations "complete, delete, edit and send to contact" for manipulate tasks on the go.

For the purpose of organize the position of these operations we used two laws of simplicity: "reduce", "organize" and "time". We applied "reduce" to show only the most important operation "complete" in the overlay showed when user wants to interact with a task. The rest of the operations were grouped (using the second law of simplicity "organize") in the detailed view of the task, which is reachable by means of the hyperlink showed in the overlay. These operations are the "delete, edit" operations, and an operation that allows user to delegate its tasks to its contacts called "send task to contact". About this operation, note that if user is prevented to perform a task because of a change in user's context, "send task to contact" provides a mechanisms for collaboration by sending tasks to others.

Furthermore, as user can navigates to a more detailed view of a selected task, the other operations are accessible by only once navigation (applying in this way the third law "time" that avoid to waste time).

Task's data. Since we follow an approach based on simplicity, we wanted different sources of information to be easy to integrate. Tasks are represented using $\mathrm{JSON}^{2}$ (JavaScript Object Notation). JSON is a widely used format in web services which are guided by the REST approximation. The use of JSON facilitated the development since it is easy to manipulate using different technologies which allows a seamlesstransition during development (e.g., from using a static text file to a task list dynamically generated by a web service).

At the prototyping stage the task dataset has been synthetically generated. It is composed by 34 tasks that provide variability in our prototype. Nevertheless, thanks to the use of JSON the integration with other task sources (e.g., services such as Remember the Milk, Google Calendar, etc.) is straightforward.

After organize requirements we determined task's attributes. These attributes represent information which is related to identification, location, transport, time, priority or completeness state. Note that each visualization shows and uses a subset of these attributes to achieve his goals.

After viewing general matters that concern all visualizations, next we detail how each visualization adapts it to the context in a non-intrusive way.

\footnotetext{
${ }^{2}$ http://www.json.org/
} 


\subsection{Map View}

Main objective. The goal of this visualization is to inform the user about the tasks that can be completed in a given location. This allows the user to plan routes, organize his mind and avoid meaningless displacements. To achieve this goal, the visualization shows a map where the user's tasks that have an assigned location are represented as markers in a geo-located way.

Adapting the visualization. For adapting the visualization according to location is important to represent the current user's location (e.g., by means of a person icon) in the screen because this inform the user where is respecting his pending tasks. Furthermore, as the underlying mobile workflow is centered in user, we focus the view on this person icon (applying the sixth law of simplicity "context").

Interacting with the Overlays. The different tasks are represented in the map by means of different markers. In order to complete tasks on the go, user selects a marker to interact with tasks geo-located in marker's position. As different tasks should be performed in the same location, markers adapt it to this situation. So, on the one hand, if exist only one task in a location, the marker only shows the representative icon of the task and has a background color according to the task's priority. On the other hand, if exists two or more task in the same location, the marker shows the number of tasks assigned to this location with a background color that depends on this number.

Regarding the interaction with a task, when a marker is selected appears an overlay with a list of task elements (see figure 2). In this overlay, we used the first law "reduce" for represent the tasks only by his name and his representative icon. We applied also the forth law "learn", taking advantage of the popular knowledge of hyperlinks. This knowledge associates an underlying text with a hyperlink. Specially, we represented the name of the task as a link to the more detailed view of the selected task. Finally, in order to "complete" a task is added a checkbox.

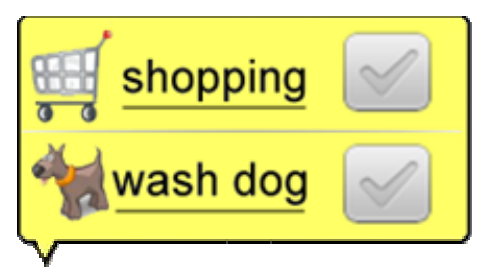

Fig. 2. Overlay example

Adding contextual zoom in and zoom out controls. The zoom controls of an application provide a general or a concrete representation of the current view. Underlying this, we think that these controls are no longer a magnifying glass. We think the zoom controls should adapt the visualization (this idea uses the sixth law "context" defining the most relevant information in the different situations, which in this case are the different values of the zoom level). So for example, user can visualize a view of its citywith its associated tasks as we see in the second image of figure 3. Next, user can use zoom out control for obtain a view of its country with his associated tasks. At this 
moment, the overlays of the tasks that are located in all neighborhoods of the city converge in only an overlay that represent the number of tasks located in the city as we can see in the first image of figure 3. On the contrary, if user is in a view of his country and focus the view on the user's city, the general overlay diverges on different overlays which are located with more accuracy. This cleans the screen of a set of markers showing the same information in a more general view.

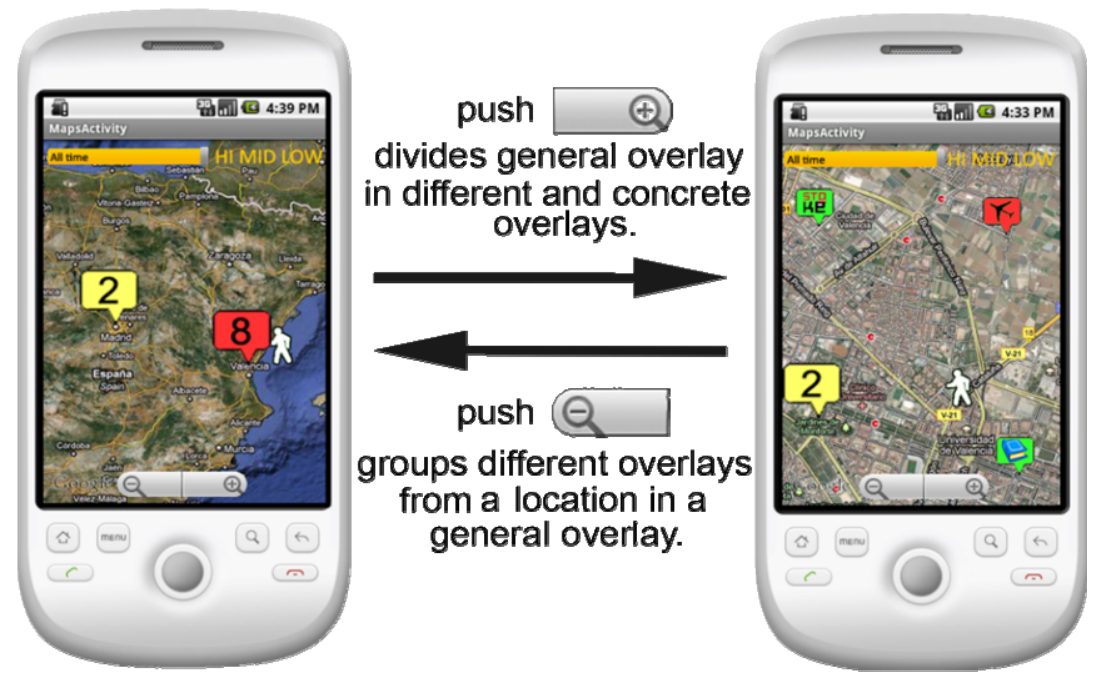

Fig. 3. View adaptation according the zoom level

\subsection{Timeline View}

Main Objective. The goalof this visualization is to provide a temporal order between the selected tasks with assigned time for its performance. This ordered vision informs the user about the next tasks that must be complete first, with associated and useful information such as temporal or economic cost or a link to the task's location for complete this.

Timeline Contents. In this visualization, the tasks are showed as a column set where each column represents one task. Therefore, applying the sixth law "context", we determine the information to be showed. So, a column shows the task's representative icon surrounded by a border that refers to its priority. Above this, its estimated duration with its associated economic cost is showed (e.g. buy a book should cost about $20 \$$ and should need about 10 minutes). In the same way, below the task icon, the visualization shows the mean of transport defined to arrive to the task's place where it must be performed with its associated time and economic cost (e.g. return home by bus costs 25 minutes and $1.5 \$$ ). Note that, by applying the first law "reduce" we hid avoid information (i.e. everybody knows that return home by foot or wash dog is for free). 
Finally, at the bottom of the screen we represent the time bar (see figure 4) that indicates the time advance, showing the tasks' times when must be performed.

\section{$\begin{array}{lllll}16: 00 h & 17: 20 h & \operatorname{mar}-30 & \text { apr- } 9 & \text { apr-19 }\end{array}$}

Fig. 4. Time bar

Adapting the visualization to the time. Since time is the most important attribute in this visualization, contextualize the view according this concern is an important requirement. Therefore, we decide first to focus the view on the current time. So, in order to inform the user which is the task that must be performed at first time we highlight the next task to be performed. Besides, for express the times of the tasks we considered the current time in order to adapt the showed information to this value. Thus, the application shows the hour when a task that must be performed is programmed for the current date. Otherwise, it shows the date where the task must be performed. This is because when a task is far from the current date, the user's mind prioritizes the date attribute against the hour attribute to organize its mind. However, for user is not relevant to visualize the date for today task since this is redundant information. The interaction design for this view is represented in figure 5.

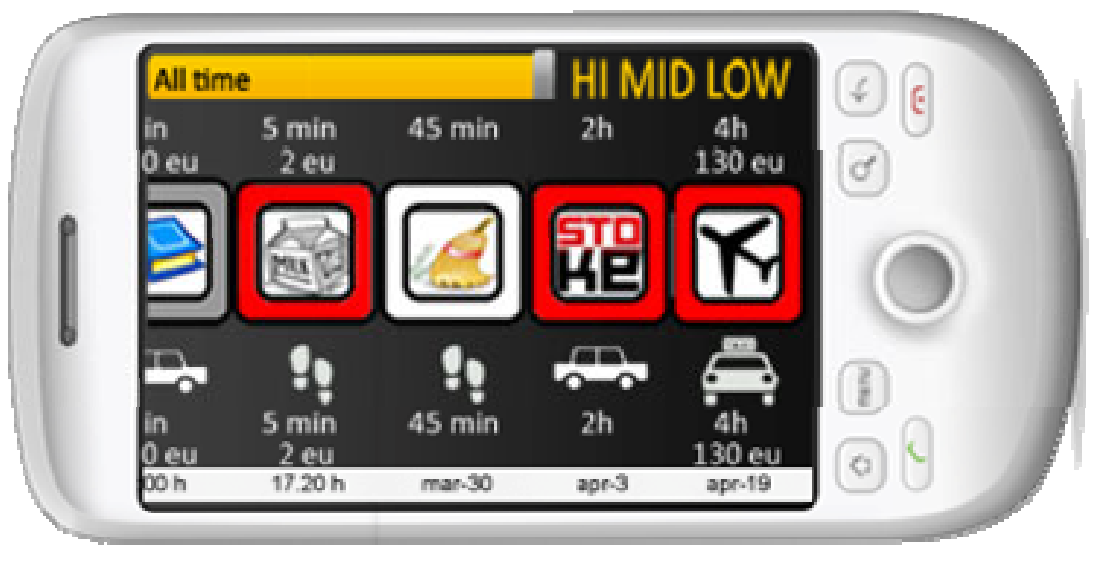

Fig. 5. Timeline View

Interacting with a task. In this view, task's interaction is different to the Map View. In this case, when user selects a task anyoverlay is showed. In this view, we associate the double-tap gesture witha link to the task's more detailed view. Moreover, if user selects the mean of transport icon is because user is thinking in a route or in how arrive to the location where task must be performed. For this purpose, applying the forth law of simplicity "learn", we link this icon to move the view to the Map View that is centered in this task's location.

Besides, in this view we take advantage of gestures in order to include the "complete" and "delete" operations in the main view. So, introducing gestural over the task icon, users can use an "up movement" when they want to complete a task and they can use a "downmovement" when they want to delete the task. 
Specifically, we propose to associate the upward movement to change the representative image of the task by a "completed" icon, and the downward movement to change the representative image of the task by a "deleted" icon. These movements may be correctedduring 5 seconds by making an opposite movement. For user, this allows a quick-useful "undo" operation for correct mistakes. However if time expired, the task is removed from the view. Note that this method of correction avoids launching out a dialog alert. For example, when user uses the negative operation "delete", we put in use the third law of simplicity "time" in order to earn user's time. In Figure 6 we see all the possible transitions occurred between these movements.

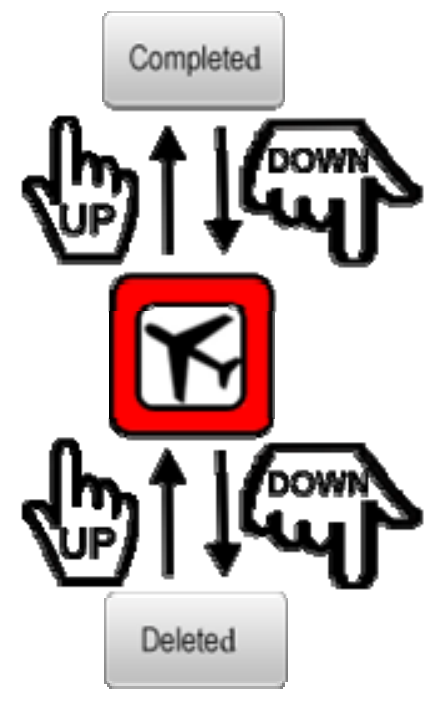

Fig. 6. Gestural interaction in Timeline View

\subsection{Cloud View}

Main objective. The main objective of this visualization is to offer a high-general view of selected tasks, where these tasks are clearly differentiated according to its priority level. It provides user with a general view of his agenda in just a screen. For this purpose, we applied the first law of simplicity "reduce" to abstract user of any other attribute such as location, time or costs. Only the task's name and task's priority are needed for a high-general view.

This visualization (see figure 7) consists in showingthe task data set as a Tag Cloud, where each tag corresponds with each task name. In order to differentiate easily the organized task by priority, the visualization uses different fonts, each one for each priority type. Thus, high priority tasks are highlighted by means of bold red color font, medium priority task are represented by means of white bold color font, and low priority tasks are showed discretely via a white no-bold font.

Interacting with a task. Interacting with a task in this view is similar with the task's interaction in Map View. When a task is selected the same overlay related in the Map 
View is presented, applying the same laws of simplicity. Furthermore, the most important operation, i.e. "complete" operation is also added in this overlay. At the same time, this selected task's font is changed for differentiate it from the rest of tags.

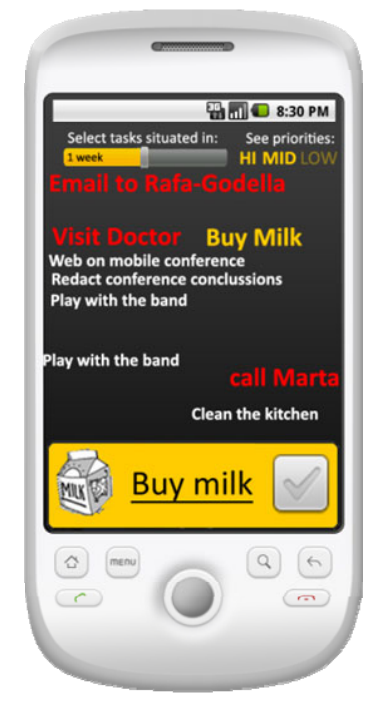

Fig. 7. Cloud View

\section{Related Work}

User Centered Design (UCD) approaches [9] stress the relevance of the user needs when designing interaction. One of the goals of UCD is to avoid the complexity introduced by technology and provide designs that fit better with the users and their activities.However, too much attention to the needs of the users can lead to a lack of cohesion and added complexity in the design [6].

UCD approaches include different steps such as specifying the context of use and requirements (e.g., by means of field studies or contextual observation), produce design solutions (e.g., prototyping) and evaluate designs (e.g., by means of field and laboratory studies). Simplicity can be considered as an implicit design goal in most of the UCD approaches, but it is not normally stated explicitly as an independent step in the process. In this work we consideredthis aspect as an explicit goal during design.

We applied the above design principles for task-based applications. A task-based application model allows users to access the services they need easily [2]. In particular, different projects exist with the goal of producing context-aware task-based applications. The Castaway project [3] stresses the relevance of location when supporting user tasks. The map view provided by Castaway has some common aspects with our map view such as the capabilities for clustering tasks. However, the Castaway does not consider the integration of different sources of contextual information. Other task-based systems such as Taskminder [4] consider time in addition to location. Taskminder represents tasks by means of a calendar and allows tasks to be set a different level of urgency and importance. Our application provides atimeline which connects task information with transport 
and cost. The use of a timeline avoids the need to provide each time period a fixed-size representation.

\section{Conclusions}

This work has provided us with guidelines for the design of context-aware task-based applications that have been useful for the design of a workflow supporting application. The next steps are: to work on our design process to define concrete functions for our simplicity-based role and to evaluate our approach through obtaining user feedback from the provided prototype outside the lab.

The developed prototype was also valuable to detect different situations where a closer integration between physical and digital worlds is required. Considering context information not only about the user but also about other people that interact with the user could help to a better organization of the user tasks. In addition, the easy specification of compensation actions for automate the handling of certain tasks also requires further study.

\section{Referentes}

[1] Giner, P., Cetina, C., Fons, J., Pelechano, V.: Developing mobile workflow support in the internet of things. IEEE Pervasive Computing 9(2), 18-26 (2010)

[2] Goth, G.: The task-based interface: Not your father's desktop. IEEE Software 26(6), 88$91(2009)$

[3] Kessell, A., Chan, C.: Castaway: a context-aware task management system. In: CHI 2006: CHI 2006 extended abstracts on Human factors in computing systems, pp. 941946. ACM, New York (2006)

[4] Landry, B.M., Nair, R., Pousman, Z., Tungare, M.: Taskminder: A context- and useraware to-do list management system. Technical report, Georgia Institute of Technology (2003)

[5] Maeda, J.: The Laws of Simplicity (Simplicity: Design, Technology, Business, Life). The MIT Press, Cambridge (2006)

[6] Norman, D.A.: Human-centered design considered harmful. Interactions 12(4), 14-19 (2005)

[7] Radi, H., Mayrhofer, R.: Towards alternative user interfaces for capturing and managing tasks with mobile devices. In: Proc. MoMM 2008: 6th International Conference on Advances in Mobile Computing and Multimedia, pp. 272-275 (November 2008)

[8] Unger, R., Chandler, C.: A Project Guide to UX Design: For user experience designers in the field or in the making. New Riders Publishing, Thousand Oaks (2009)

[9] Vredenburg, K., Mao, J.-Y., Smith, P.W., Carey, T.: A survey of user-centered design practice. In: CHI 2002: Proceedings of the SIGCHI conference on Human factors in computing systems, pp. 471-478. ACM, New York (2002)

[10] Dey, A.K., Abowd, G.D.: Towards a Better Understanding of Context and ContextAwareness. In: CHI 2000 Workshop on the What, Who, Where, When, and How of Context-Awareness (2000) 